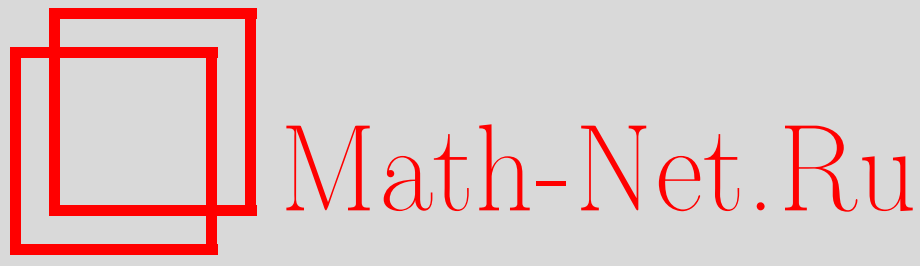

Обцероссийский математический портал

Н. Т. Тлеуханова, О преобразовании Харди и Беллмана для ортогональных рядов Фурье, Матем. заметки, 2001, том 70, выпуск 4, 638-640

DOI: https://doi.org/10.4213/mzm776

Использование Общероссийского математического портала Math-Net.Ru подразумевает, что вы прочитали и согласны с пользовательским соглашением http://www.mathnet.ru/rus/agreement

Параметры загрузки:

IP: 3.89 .185 .249

26 апреля 2023 г., 15:48:26 


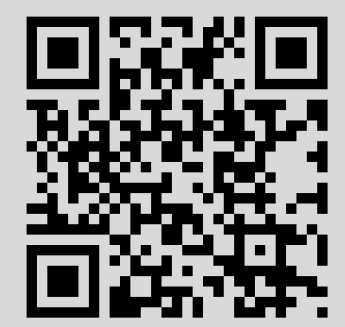




\section{О ПРЕОБРАЗОВАНИИ ХАРДИ И БЕЛЛМАНА ДЛЯ ОРТОГОНАЛЬНЫХ РЯДОВ ФУРЬЕ}

\section{Н. Т. Тлеуханова}

Хорошо известны результаты Харди и Беллмана [1], [2] для тригонометрических рядов Фурье: если $f \in L_{p}[0, \pi]$,

$$
f \sim \sum_{k=0}^{\infty} a_{k} \cos k x, \quad A_{k}=\frac{1}{k+1} \sum_{m=0}^{k} a_{m}, \quad B_{k}=\sum_{m=k}^{\infty} \frac{a_{m}}{m},
$$

то ряды

$$
\sum_{k=0}^{\infty} A_{k} \cos k x, \quad \sum_{k=1}^{\infty} B_{k} \cos k x
$$

являются рядами Фурье некоторых функций $H f, B f$ из $L_{p}[0, \pi]$ и верны оценки

$$
\|H f\|_{p} \leqslant c\|f\|_{p}, \quad\|B f\|_{p} \leqslant c\|f\|_{p} .
$$

Данные преобразования называют преобразованиями Харди и Беллмана.

В заметке рассматриваются обобщения этих результатов в трех направлениях: рассматриваются ряды Фурье относительно регулярных ортонормированных систем [3] (охватывающие все тригонометрические и мультипликативные системы с ограниченными образующими); вводится обобщение преобразований Харди и Беллмана; изучается ограниченность введенных преобразований в анизотропных пространствах Лоренца [3].

ОПРЕДЕЛЕНИЕ 1. Ортонормированную систему $\Phi=\left\{\phi_{k}(x)\right\}_{k=1}^{\infty}$ назовем регулярной, если существует константа $B$ такая, что

1) для любого отрезка $e$ из $[0,1]$ и $k \in \mathbb{N}$ верна оценка

$$
\left|\int_{e} \phi_{k}(x) d x\right| \leqslant B \min \left(\mu e, \frac{1}{k}\right) ;
$$

2 ) для любого отрезка $w$ из $\mathbb{N}$ (конечная арифмеетическая прогрессия с шагом 1$)$ и $t \in(0,1]$ выполнена оценка

$$
\left(\sum_{k \in w} \phi_{k}(f)\right)^{*}(t) \leqslant B \min \left(|w|, \frac{1}{t}\right)
$$


где $\left(\sum_{k \in w} \phi_{k}(f)\right)^{*}(t)$ - невозрастающая перестановка функции $\sum_{k \in w} \phi_{k}(x),|w|-$ количество элементов в множестве $w$.

Пусть $\Phi$ - регулярная ортонормированная система, $I=\left\{I_{k}\right\}_{k \in \mathbb{N}}-$ некоторая последовательность конечных подмножеств из $\mathbb{N}$. Через $J$ обозначим последовательность множеств $\left\{J_{k}\right\}_{k \in \mathbb{N}}$, где $J_{k}=\left\{m: k \in I_{m}\right\}$. Для $f \in L_{1}[0,1], f \sim \sum_{k=1}^{n} a_{k} \varphi_{k}(x)$, и последовательности $I=\left\{I_{k}\right\}_{k \in \mathbb{N}}$ определим преобразования $H(f ; I)$ и $B(f ; I)$ следуюшим образом:

$$
\begin{aligned}
& H(f ; I) \sim \sum_{k=1}^{\infty} \frac{1}{\left|I_{k}\right|}\left(\sum_{m \in I_{k}} a_{m}\right) \varphi_{k}(x), \\
& B(f ; I) \sim \sum_{k=1}^{\infty}\left(\sum_{m \in J_{k}} \frac{a_{m}}{\left|I_{m}\right|}\right) \varphi_{k}(x) .
\end{aligned}
$$

Назовем их соответственно преобразованиями Харди и Беллмана, отвечающими последовательностям множеств $I=\left\{I_{k}\right\}_{k \in \mathbb{N}}$. В случае $I_{k}=\{1,2, \ldots, k\}, k \in \mathbb{N}$, эти преобразования являются соответственно преобразованиями Харди и Беллмана.

Через $L_{p q}[0,1]$ обозначим пространство Лоренца, а через $l_{p q}$ - дискретное пространство Лоренца.

Tеорема 1. Пусть $2<p<\infty, p^{\prime}=p /(p-1), 1 \leqslant q \leqslant \infty, \Phi=\left\{\varphi_{k}(x)\right\}_{k=1}^{\infty}-$ pегулярная

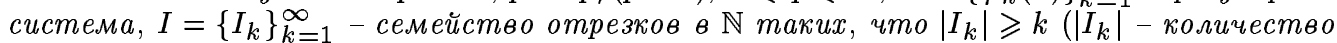
әлементов в $\left.I_{k}\right)$. Тогда преобразования Харди $H(f, I)$ и Беллмана $B(f, I)$ ограничены $в$ пространствах $L_{p q}[0,1]$ и $L_{p^{\prime}}[0,1]$ соответственно, т.е.

$$
\|H(f, I)\|_{L_{p q}} \leqslant c\|f\|_{L_{p q}}, \quad\|B(f, I)\|_{L_{p^{\prime} q}} \leqslant c\|f\|_{L_{p^{\prime} q}} .
$$

Tеорема 2. Пусть $1<p \leqslant 2, p^{\prime}=p /(p-1), 1 \leqslant q \leqslant \infty, \Phi=\left\{\varphi_{k}(x)\right\}_{k=1}^{\infty}-$ pezуляpная система, $I=\left\{I_{k}\right\}_{k=1}^{\infty}-$ семейство конечных множеств из $\mathbb{N}$, удовлетворяти, их условиям $\left|I_{k}\right|=k$ и $I_{k} \subset I_{k+1}$. Тогда преобразования Харди $H(f, I)$ и Беллмана $B(f, I)$ ограничены в пространствах $L_{p q}[0,1]$ u $L_{p^{\prime} q}[0,1]$ соответственно.

Tеорема 3. Пусть $2<p<\infty, 1 \leqslant q \leqslant \infty, \Phi=\left\{\varphi_{k}(x)\right\}_{k=1}^{\infty}-$ регулярная система, $\left\{Q_{t}\right\}_{t \in(0,1)}$ - некоторое семейство отрезков из $[0,1]$, удовлетворяющ, их условию $\mu Q_{t} \geqslant t$. Если $f \sim \sum_{k=1}^{\infty} a_{k} \varphi_{k}(x)$ и $a=\left\{a_{k}\right\}_{k=1}^{\infty} \in l_{p q}$, то последовательность коэффичиентов фурье $b=\left\{b_{k}\right\}_{k=1}^{\infty}$ функиии

$$
F(t)=\frac{1}{\mu Q_{t}} \int_{Q_{t}} f(x) d x
$$

также принадлехит пространству Лоренца $l_{p q}$ и верно неравенство

$$
\|b\|_{l_{p q}} \leqslant c\|a\|_{l_{p q}} .
$$

Tеорема 4. Пусть $1<p<2,1 \leqslant q \leqslant \infty, \Phi=\left\{\varphi_{k}(x)\right\}_{k=1}^{\infty}-$ регулярная система, $f \sim \sum_{k=1}^{\infty} a_{k} \varphi_{k}(x)$ и $a=\left\{a_{k}\right\}_{k=1}^{\infty} \in l_{p q}, g-$ произвольная, равноизмеримая с $f$ функиия. Тогда последовательность коэффичиентов Фурье $b=\left\{b_{k}\right\}_{k=1}^{\infty}$ функиии

$$
F(t)=\frac{1}{t} \int_{0}^{t} g(x) d x, \quad t \in(0,1),
$$

такхе принадлехит пространству Лоренца $l_{p q}$ и верно неравенство

$$
\|b\|_{l_{p q}} \leqslant c\|a\|_{l_{p q}} .
$$

Пусть $\boldsymbol{p}=\left(p_{1}, \ldots, p_{n}\right), \boldsymbol{q}=\left(q_{1}, \ldots, q_{n}\right)$ - векторы такие, что если $0<q_{j}<\infty$, то $0<p_{j}<\infty$, если же $q_{j}=\infty$, то $0<p_{j} \leqslant \infty, j=1, \ldots, n$. 
Анизотропное пространство $L_{\boldsymbol{p q}}[0,1]^{n}$ определяется как множество измеримых функций $f$, для которых

$$
\|f\|_{L_{\boldsymbol{p q}}}=\left(\int_{0}^{1} \cdots\left(\int_{0}^{1}\left|t_{1}^{1 / p_{1}} \cdots t_{n}^{1 / p_{n}} f^{*_{1}, \ldots, * n}\left(t_{1}, \ldots, t_{n}\right)\right|^{q_{1}} \frac{d t_{1}}{t_{1}}\right)^{q_{2} / q_{1}} \cdots \frac{d t_{n}}{t_{n}}\right)^{1 / q_{n}}<\infty
$$

где $f^{* 1}, \ldots, *_{n}\left(t_{1}, \ldots, t_{n}\right)$ - невозрастающая перестановка функции $f$, взятая последовательно по переменным $x_{1}, \ldots, x_{n}$ (считая остальные переменные фиксированными), выражение

$$
\left(\int_{0}^{1}(F(t))^{q} \frac{d t}{t}\right)^{1 / q}
$$

при $q=\infty$ понимается как

$$
\sup _{t>0} F(t)
$$

Пусть $\Psi_{1}=\left\{\psi_{k}^{1}(x)\right\}_{k=1}^{\infty}, \ldots, \Psi_{n}=\left\{\psi_{k}^{n}(x)\right\}_{k=1}^{\infty}-$ регулярные системы функций. Определим $\Phi=\left\{\phi_{k}(x)\right\}_{k \in \mathbb{N} n}$ следуюшим образом:

$$
\phi_{k}(x)=\phi_{k_{1} \ldots k_{n}}\left(x_{1}, \ldots, x_{n}\right)=\psi_{k_{1}}^{1}\left(x_{1}\right) \cdots \psi_{k_{n}}^{n}\left(x_{n}\right) .
$$

Пусть $I_{p}=\left\{I_{k}^{p}\right\}_{k \in \mathbb{N} n}$ - некоторое семейство конечных подмножеств $\mathbb{N}$, удовлетворяющих условию: при $p>2$ семейство $I_{p}$ совпадает с множеством отрезков $\left\{I_{k}^{p}\right\}_{k \in \mathbb{N} n}$ таких, что $\left|I_{k}^{p}\right| \geqslant k$, a при $p \leqslant 2 I_{p}$ есть семейство множеств $\left\{I_{k}^{p}\right\}_{k \in \mathbb{N} n}$ таких, что $I_{k}^{p} \subset I_{k+1}^{p}$ и $\left|I_{k}^{p}\right|=k$.

Пусть $1<p=\left(p_{1}, \ldots, p_{n}\right)<\infty, f \sim \sum_{k \in \mathbb{N}^{n}} a_{k} \phi_{k}(x)$. Определим преобразования $B_{j}\left(f, I_{p_{j}}\right)$ и $H_{j}\left(f, I_{p_{j}}\right)$ следующим образом:

$$
\begin{aligned}
& H_{j}\left(f, I_{p_{j}}\right)=\sum_{k_{1}=1}^{\infty} \cdots \sum_{k_{n}=1}^{\infty} \frac{1}{\left|I_{k_{j}}^{p_{j}}\right|}\left(\sum_{m_{j} \in I_{k_{j}}^{p_{j}}} a_{k_{1}, \ldots, m_{j}, \ldots, k_{n}}\right) \phi_{k_{1} \ldots k_{n}}\left(x_{1}, \ldots, x_{n}\right), \\
& B_{j}\left(f, I_{p_{j}}\right)=\sum_{k_{1}=1}^{\infty} \cdots \sum_{k_{n}=1}^{\infty}\left(\sum_{m_{j} \in J_{k_{j}}^{p_{j}}} \frac{a_{k_{1}, \ldots, m_{j}, \ldots, k_{n}}}{\left|I_{m_{j}}^{p_{j}}\right|}\right) \phi_{k_{1} \ldots k_{n}}\left(x_{1}, \ldots, x_{n}\right) .
\end{aligned}
$$

Пусть $E=\left\{\varepsilon=\left(\varepsilon_{1}, \ldots, \varepsilon_{n}\right): \varepsilon_{i}=0\right.$ или $\left.\varepsilon_{i}=1\right\}$ - вершины $n$-мерного единичного куба, $G_{p}=I_{p_{1}} \times \cdots \times I_{p_{n}}=\left\{I_{k_{1}}^{p_{1}} \times \cdots \times I_{k_{n}}^{p_{n}}\right\}_{k \in \mathbb{N}^{n}}$. Оператор $T_{\varepsilon}\left(f, G_{p}\right), \varepsilon \in E$, определим следующим образом:

$$
T_{\varepsilon}\left(f ; G_{p}\right)=T_{\varepsilon_{n}} \cdots T_{\varepsilon_{1}}(f)
$$

где

$$
T_{\varepsilon_{j}}(f)=\left\{\begin{array}{l}
H_{j}\left(f ; I_{p_{j}}\right), \text { при } \varepsilon_{j}=1, \\
B_{j}\left(f ; I_{p_{j}^{\prime}}\right), \text { при } \varepsilon_{j}=0, p^{\prime}=\frac{p}{p-1} .
\end{array}\right.
$$

Tеорема 5. Пусть $1<\boldsymbol{p}=\left(p_{1}, \ldots, p_{n}\right)<\infty, 1 \leqslant \boldsymbol{q}=\left(q_{1}, \ldots, q_{n}\right) \leqslant \infty, E-$ вериинь единичного куба, $G_{p}=I_{p_{1}} \times \cdots \times I_{p_{n}}$. Тогда для любого $\varepsilon \in E$ верно неравенство

$$
\left\|T_{\varepsilon}\left(f ; G_{p}\right)\right\|_{L_{p q}} \leqslant c\|f\|_{L_{p q}} .
$$

\section{СПИСОК ЦИТИРОВАННОЙ ЛИТЕРАТУРЫ}

1. Hardy G.H. // Messages of Math. 1958. V. 58. P. 50-52. 2. Bellman R. // Bull. Amer. Math. Soc. 1944. V. 50. № 10. Р. 741-744. 3. Нурсултанов Е. Д. // Изв. РАН. Сер. матем. 2000. T. 64. №1. C. $95-122$. 